\title{
Random Walk and Graph Cut for Co-Segmentation of Lung Tumor on PET-CT Images
}

\author{
Wei Ju, Deihui Xiang, Bin Zhang, Lirong Wang, Ivica Kopriva Senior Member IEEE, and Xinjian \\ Chen*, Senior Member IEEE
}

\begin{abstract}
Accurate lung tumor delineation plays an important role in radiotherapy treatment planning. Since the lung tumor has poor boundary in PET images and low contrast in CT images, segmentation of tumor in PET and CT images is a challenging task. In this study, we effectively integrate the two modalities by making fully use of the superior contrast of PET images and superior spatial resolution of CT images. Random walk and graph cut method are integrated to solve the segmentation problem, in which random walk is utilized as an initialization tool to provide object seeds for graph cut segmentation on PET and CT images. The co-segmentation problem is formulated as an energy minimization problem which is solved by max-flow/min-cut method. A graph includes two sub-graphs and a special link is constructed, in which one sub-graph is for PET and another is for CT, and the special link encodes a context term which penalizes the difference of the tumor segmentation on the two modalities. To fully utilize the characteristics of PET and CT images, a novel energy representation is devised. For PET, a downhill cost and a 3D derivative cost is proposed. For CT, a shape penalty cost is integrated into the region and boundary function which helps constrain the tumor location during the segmentation. We validate our algorithm on a dataset which consists of 18 PET-CT images. The experimental results indicate the proposed method is superior to the graph cut method solely using the PET or CT is more accurate compared with the random walk method, random walk co-segmentation method, and non-improved graph-cut method.
\end{abstract}

Index Terms-Image segmentation, interactive segmentation, graph cut, random walks, prior information, lung tumor, positron emission tomography (PET), computed tomography (CT).

This work was supported in part by the National Basic Research Program of China (973 Program) under Grant 2014CB748600, in part by Natural Science Foundation of the Jiangsu Province under Grant BK20140052, and in part by the National Natural Science Foundation of China under Grant 81371629, Grant 61401293, Grant 61401294, Grant 81401451, and Grant 81401472. The. W. Ju, D. Xiang, L. Wang, and X. Chen are with the School of Electronic and Information Engineering, Soochow University, Suzhou 215123, China (e-mail: yehesu@163.com; xiangdehui@suda.edu.cn; wanglirong@ suda.edu.cn; xjchen@suda.edu.cn).(Wei Ju and Deihui Xiang contributed equally to this work.) B. Zhang is with the First Affiliated Hospital of Soochow University, Suzhou 215123, China (e-mail: zbnuclmd@126.com). I. Kopriva is with the Division of Laser and Atomic Research and Development, Ruder Bošković Institute, Zagreb 10000, Croatia (e-mail: ikopriva@irb.hr). * Corresponding author

\section{INTRODUCTION}

Accurate tumor segmentation plays a significant role in image-guided radiation therapy. Due to the variability and diversity of medical images, it is a non-trivial and challenging task for cancer assessment and treatment planning [1], [2]. For effective cancer assessment in clinic, Positron Emission Tomography (PET) and Computed Tomography (CT) are two commonly used imaging technologies. In PET images, the diseased areas, such as tumor and inflammation, appear as 'hot' areas reflecting high contrast to the normal surrounding tissues. The high contrast in PET images makes it easy to distinguish the malignant areas from the normal tissues and gives the observer a distinct visualization which reduces the observer variability [3]. However, due to the low spatial resolution of PET, the target boundary definition is poor and fuzzy [see Fig.1(a)]. Thus accurate tumor segmentation using PET only is problematic. CT images have high spatial resolution and provide detailed anatomical information, yet lacking metabolism information. Lack of physiological information in CT makes it difficult to distinguish between the lesion and the normal tissues because the tumor intensity is similar to the surrounding tissues [5] [see Fig.1(b)]. Many studies indicated that the combination of PET and CT images produce a more consistent tumor volumes [6]-[8]. Therefore, PET-CT gained a lot of attention in both clinic and the field of image processing and has become a standard method for tumor delineation and cancer assessment.

Although PET-CT images have been widely used in clinic, automatic segmentation on PET-CT images is still a challenging task. The existing methods working for PET-CT [10]-[13] have an underlying assumption that the tumor volume is identical on PET and CT, attempting to get one tumor contour from PET and CT two modalities. However, as PET and CT convey different information which is not always complementary, but sometimes contradictory, the tumor volume may be different on PET and CT [14]. In this work, we strive to segment tumor volumes simultaneously by taking the advantages from two modalities, the superior contrast of PET images and superior spatial resolution of CT images. Two different tumor volumes are obtained, the volumes defined on PET and the tumor contours defined on CT. Our method is based on Boykov's graph cut method [15]-[19] and Leo Grady's random walk method [20], [21]. Both of the methods treat the image as a graph and minimize energy functions on the 

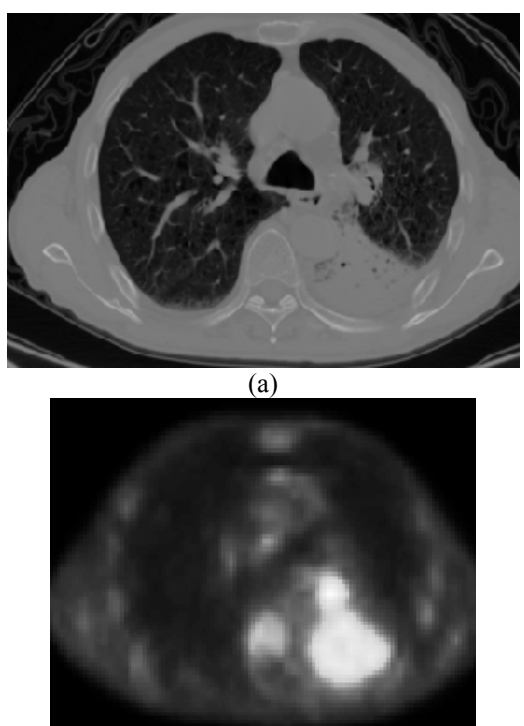

(b)

Fig. 1. PET and CT images. (a) One slice of a CT image. (b) The corresponding PET image.

constructed graph to produce an optimal segmentation. In both methods, a weighted graph is constructed. Nodes of the graph correspond to voxels in image and edges are placed between nearby voxels [22]. The edge weights are determined by the character of the image intensity. We incorporate the two methods together by utilizing random walk as an initial preprocessor and graph cut as a co-segmentation problem solver. Random walk is utilized to provide initial lesion detection on PET which will be used as object seed sets for PET and $\mathrm{CT}$ and also as an initial shape model for tumors in CT images. The co-segmentation problem is formulated as an energy minimization problem. As a graph-based method, the performance of the graph cut method is largely controlled by the energy function. We proposed a novel energy function which is well adjusted to the characteristic of PET and CT images. The energy function for both PET and CT segmentation has two terms, region term and boundary term. For PET, the region cost consists of three components, a cost based on SUV distribution, a downhill cost and a three-dimensional (3D) derivate function. The downhill cost is based on the analysis of tumor uptake. The $3 \mathrm{D}$ derivative feature is formulated using the Hessian matrix combined with Gaussian functions. For CT, a shape constraint term is integrated into the region cost function. To get consistent results between PET and CT, a context term which penalizes the difference between them is added to the energy. The optimal solution with respect to energy function minimization will be achieved in a polynomial time by computing maximum flow in the constructed graph.

The rest of the paper is organized as follows. In section II, we give a brief review on tumor segmentation using PET and CT images and characterize the novelty of our approach. In section III, we describe the framework of our method and give a detailed introduction of random walk and graph cut method, including the description of the energy function design. Section IV gives detailed description of the experiments and presents the experimental results. Section V, we discuss our work and the future improvement. Finally, Section VI draws the conclusion.

\section{RELATED WORK}

PET and CT images have been actively in use for target delineation in clinical radiotherapy applications. For PET segmentation, the most widely used is thresholding method which may use clinically experienced fixed value (i.e., $S U F$ (Standardized uptake value) $=2.5$ ) or a percentage of the maximum SUP [23] (i.e. 50\% of $5 U F_{M A X}$ ) as thresholds. Based on the thresholding techniques, some approaches, such as Fuzzy-C-Means (FCM) [27], Fuzzy Locally Adaptive Bayesian (FLAB) [28] and iterative thresholding methods (ITM)[29] which has good performance in dealing with indistinct nature of tumor boundary have been extensively developed.

Recently, graph based methods have attracted a lot of attention due to its good performance in image segmentation. Ulas et al. [30] demonstrated the effectiveness of segmenting lung tumor on PET images using random walk algorithm. Cherry et al. [31] showed how to extract heart, liver and regions effectively which have similar uptake value to lesions by merging a novel monotonic downhill function with the conventional graph cut energy regularization.

However, these methods only conduct on single modality. As physically limited spatial resolution and lack of anatomical information on PET images, many developers recently attempt

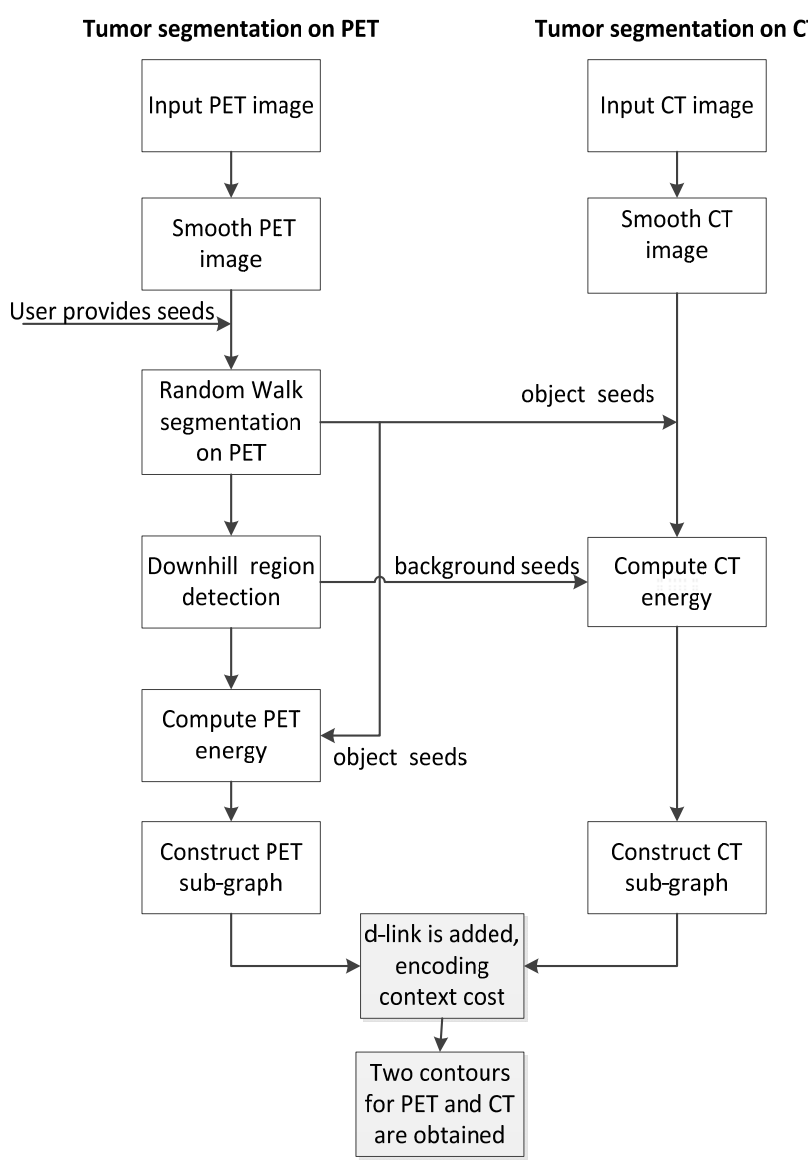

Fig. 2. Flowchart of the algorithm 
to integrate PET and CT images, striving to achieve more precise lesion detection and delineation. Ulas et al. [10] proposed an automatic random-walk-based segmentation method combining PET, CT and MRI three modalities by the hyper graph construction. Han [12] developed an optimal graph-based co-segmentation method with the establishment of an arbitration graph mechanism, encoding the characterized information of PET and CT images and the context information between them. Based on Han's method, Qi [14] reduced the size of the constructed graph in Han's paper from three sub-graphs to two and tried to obtain two different tumor contours on PET and CT.

Our work is motivated by Qi's method [14]. We both formulate the co-segmentation problem as an optimal labeling of Markov Random Field (MRF) on the constructed graph with PET and CT images. Compared to Qi's method, the innovation in our work is the combination of random walk and graph cut and the introduction of the novel energy representation which is introduced as above. We utilize random walk technique to provide initial hot spot detection which is treated as seed points required by Graph cut. As far as common knows, graph cut algorithm has small cut and shrinking problem in noisy images if a small number of seeds are used. The prior tumor appearance provided by random walk will avoid the small cut problem happening and reduce the user's intervention. Since random walk is more efficacious in handling ambiguities among object boundaries, the initial detection by random walk will facilitate the accurate identification of target regions for co-segmentation on PET-CT images. Moreover, the novel feature terms we integrated into the energy function have great significance in segmenting tumor from PET and CT. For PET segmentation, the downhill term helps to identify the location of the tumor and the $3 \mathrm{D}$ derivative feature enhances the tumor structures and weakens the background field, which all has contribution to producing accurate lesion segmentation. For CT segmentation, shape constraint gives a penalty to the region outside the tumor model area and helps to constrain the tumor region.

\section{METHODS}

Our proposed algorithm is based on two graph-based methods, random walk (RW) and graph cut (GC). Random walk is an initial preprocessor for PET, providing basic tumor appearance which is used as seed points for PET and CT. Then the task of simultaneous segmentation is formulated as an energy minimization problem on graphs corresponding to PET and CT images. The graph is constructed involving two sub-graphs, one for PET and one for CT. Inter-graph arcs called $d$-link are added to connect correspondent nodes of the two sub-graphs. For each sub-graph, a novel energy expression is designed. For PET, the weight of arcs encodes region and boundary information. The region cost function consists of three different kinds of feature extracted from PET images, including downhill feature, 3D derivative feature and SUV distribution feature. The downhill feature based on the analysis of tumor characteristics in PET would help extract the ambiguous area which has a similar intensity distribution to the tumor such as heart and liver. The 3D derivative feature is able to enhance the tumor objects. The SUV distribution feature
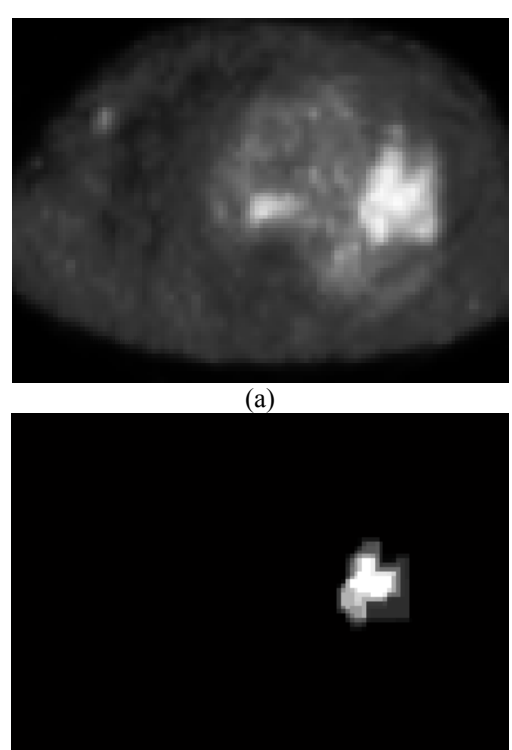

(b)

Fig. 3. One typical slice of the probabilities at each voxel that a random walker released from that voxel reaches to the foreground seed. (a) original PET image (b) the corresponding image with the probabilities

helps to solve the problem of heterogeneity in PET images. For CT segmentation, a shape penalty term is incorporated with region and boundary information. The inter-graph arcs enforce context information between PET and CT, enabling target contours to follow salient features of each modality [14].

\section{A. Random walk for tumor segmentation on PET images}

Random walk, a kind of graph-based segmentation approach, is an effectively interactive method. In this section, we give a detailed introduction of random walk and its use in initial tumor segmentation on PET images.

Assume a graph $G$ is connected and undirected, we represent graph $G$ as $G=(\nabla, E)$ with vertices $\forall \in V$ and edges $E X V Y . e_{i j}$ is an edge connecting node $v_{i}$ and its neighborhood $v_{j}$ with a weight $w_{i j}$. The vertices in the graph can be divided into two sets, $\nabla_{M}$ and $\nabla_{V}$, such that $\nabla_{M} \cup \nabla_{V}=\nabla$ and $\nabla_{M} \cap \nabla_{V}=\emptyset \cdot V_{M}$ is a labeling seed set which is defined by users and $V_{V}$ contains all unknown nodes. The segmentation problem is to find appropriate labeling for the unknown nodes. A weighting function maps a change in image intensity to edge weight corresponding to the probability of the random walker first reaches a seed point. The weighting function we employed is the typical Gaussian function derived from the suggestion in (Grady, 2006) [20].

$$
w_{q j}=\exp \left(-\beta(g i-g)^{2}\right)
$$

where $g_{i}$ indicates the intensity at voxel $i$, the value $\beta$ represents weighting parameter. It has been demonstrated previously that the desired probabilities the random walker passes through the edge has the same solution as the combinatorial Dirichlet problem. The formulation of Dirichlet integral is stated as: 


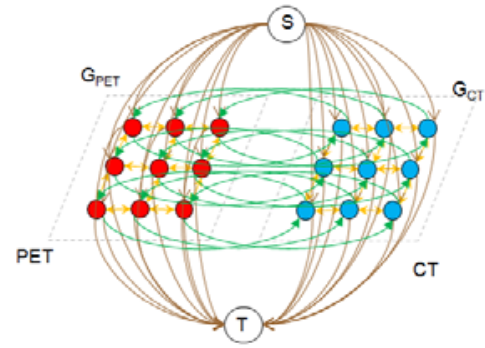

Fig.4. The constructed graph with two sub-graphs $\mathbf{G}_{\mathbf{P E}}$ and $\mathbf{G}_{\mathbf{G T}}$ and d-link arcs encoding the context penalties

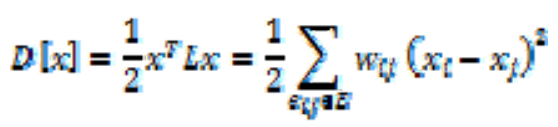

where $x$ denotes the probability at each voxel, $L$ represents the combinatorial Laplacian matrix. The matrix is defined as:

$$
E_{q}=\left\{\begin{array}{cl}
d_{l} & \text { if } t=f_{t} \\
-w_{t} & \text { if } v_{l} \text { and } v_{j} \text { are adjacent nades, } \\
0 & \text { atherwise }
\end{array}\right.
$$

where $L_{i j}$ is indexed by vertices $v_{i}$ and $v_{j}, d_{i}$ is the degree of a vertex for all edges $\theta_{i j}$ incident on $v_{i}$ and has the definition as: $d_{l}=\Sigma w\left(\sigma_{t}\right)$.

\section{B. Graph cut for co-segmentation of tumor on PET-CT images}

In this section, we will introduce how to segment tumor concurrently on PET and CT images using graph cut technique. The rationale is to formulate the segmentation problem as finding the optimal labeling by energy minimization. In this paper, graph cut is automatic without user's guidance of the desired regions to be segmented. The initial lesion appearance detected by random walk and the feature of tumor adjusted to the PET energy function will facilitate the identification of the object and background seeds. The optimal solution will be achieved by solving a maximum flow problem in low-order polynomial time.

\section{Graph construction}

Two sub-graphs $G_{\mathrm{PEF}}$ for PET, $G_{\mathrm{Cr}}$ for CT, and a connector which is called d-link constitute the whole graph. Each sub-graph contains n-links and t-links, encoding the neighboring costs and regional costs. $d$-links enforce the context information between PET and CT. The graph is shown in Fig.4. For each voxel $u \in G_{\mathrm{PEF}}, u^{x} \in G_{\mathrm{EF}}$ is the corresponding voxel in CT. The intensity value for voxel $u$ is noted by $g_{u}$. Label $f$ indicates that the voxel belongs to the target object $(f=1)$ or to the background $(f=0)$. The energy term for each sub-graph PET and CT is expressed as $E_{F E T}$ and $E_{C T}$. $E_{\text {sontext: }}$ denotes the context cost penalizing the difference between PET and CT. The total cost function is defined in the following way.

$$
E\left(f^{\prime}\right)=E_{R E T}\left(f^{\prime}\right)+E_{E F}\left(f^{\prime}\right)+E_{\text {sontext }}(f)
$$

Our goal is to minimize the energy such that the optimal segmentation of PET-CT images is attained.

\section{Cost function for PET}

To introduce our energy function, we start with the expression of PET cost function. The cost has a region term consisting of a SUV cost, a downhill cost and a 3D derivative cost and a boundary term. The SUV term $\mathbb{F}_{u}\left(f_{w}\right)$ is based on the SUV distribution of PET images. The downhill term $D_{u}\left(f_{u}\right)$ is adjusted to the downhill feature of tumor on PET and the 3D derivative cost $F_{u}(f)$ utilizes the derivative feature of PET images. The boundary term $B_{w v}(f, f)$ is based on the gradient of the image. The energy function for the segmentation of PET is defined as follows.

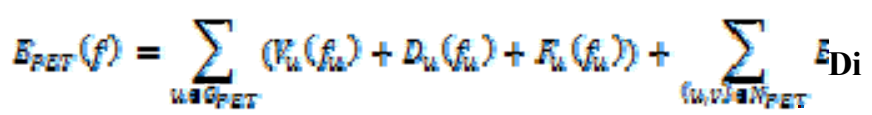

where $\hat{H}_{\text {FET }}$ denotes the neighboring relationship between voxels in PET graph $G_{\mathrm{PET} T}$. The detailed definition of every function term is presented as follows.

1) Region term

The region term $\mathbb{V}_{u}\left(f_{W}\right)$ is the likelihood assigning label $f_{u}$ to the voxel $u$. Instead of user identifying object seeds, the pre-segmented tumor appearance (which is conducted by random walk) is considered as target regions desired to be segmented. Therefore, for every voxel inside the pre-segmented field, $\nabla_{u}\left(f_{u}=1\right)=0$ and $F_{u}\left(f_{u}=0\right)=+\infty$. In this paper, there is no need to define the background seed sets for PET segmentation because a downhill cost which we defined can be able to extract the ambiguous area which has the similar SUV value to the tumor. For the spheres outside the initial identified region, it is important to note that the tumor may contain regions of necrosis or cystic change such that FDG uptake throughout the tumor is heterogeneous. To solve the problem of heterogeneity, we design the cost function based on the prior knowledge and the feature of SUV distribution of tumor. Based on prior knowledge, it is highly possible that the voxels having SUV value higher than $50 \%$ of maximum SUV belong to the tumor. We denote $S_{W A X}$ the maximum SUV value. For every voxel with a lower value than $15 \%$ of $S_{\text {MAXX }}$, it is more likely belonging to the background. The function is defined as:

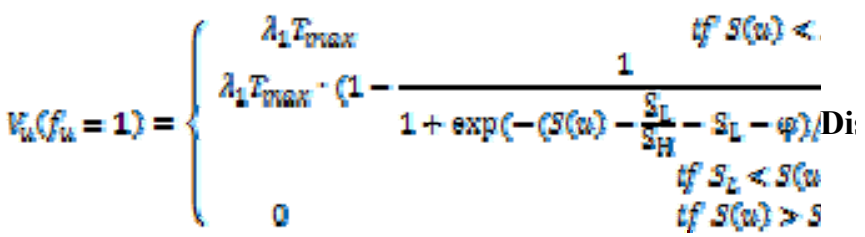



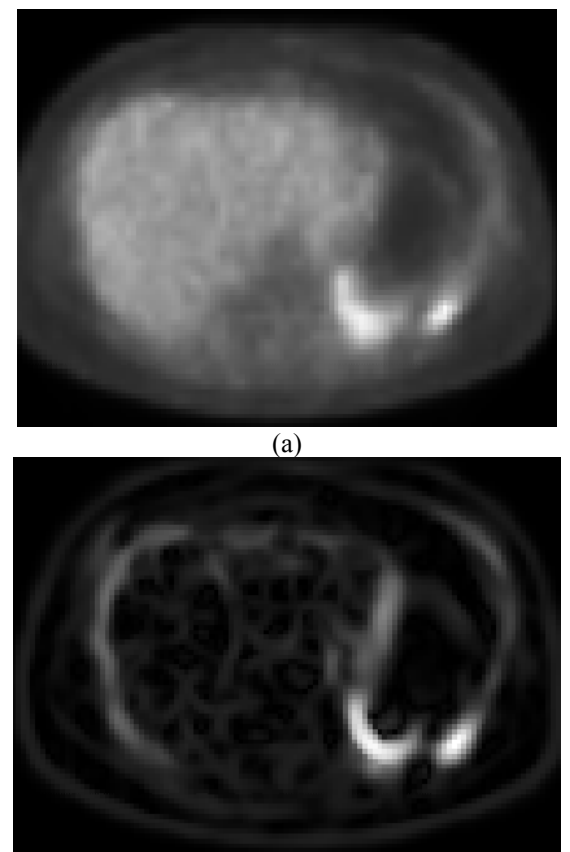

(b)

Fig. 5. one example slice of a 3D derivative feature image (a) original PET image (b) 3D derivative feature image. The image shows that the heart area with high uptake value which is similar to the tumor SUV uptake is weakened and the lesions are enhanced.

$$
F_{u}\left(f_{u}=0\right)=\lambda_{1} T_{\max }-F_{u}\left(f_{u}=1\right)
$$

where $T_{\max }$ is the maximum region cost allowed; $S_{H}=50 \% \cdot S_{M A X}$ and $S_{L}=15 \% \cdot S_{M A X}$ are the higher and lower threshold value. $\gamma$ is the parameter controlling the curvature of the function. $\varphi$ controls the center point of the function. $\lambda_{1}$ is the scaling constant which maintains the role of the SUV cost. We employ the function instead of the linear function because it is monotonically increasing, more versatile and more robustness to the heterogeneity.

\section{2) 3 D Derivative term}

The $3 \mathrm{D}$ derivative cost is a function using three-dimensional derivative features which is calculated from a tumor volume as well as the volume intensity [31], [32]. The basic idea is to characterize the tissue not only on its intensity values but also its local intensity structures. Although the shape of tumor is irregular, the approximate tumor volume which is a nodule can be regarded as a blob-like structure. The 3D filter we employed here can effectively respond to specific blob-like objects. The three-dimensional filter is based on the gradient vector and Hessian matrix of the volume intensity function combined with isotropic Gaussian functions.

Let $\boldsymbol{g}(\boldsymbol{x})$ be an intensity function of an image, where $\mathbf{x}=(x, y, z)$. The characteristic of structures can be analyzed using the second-order approximation of $g(x)$. The second-order structures can be formulated as follows:

$g^{x}(x)=g\left(\mathbf{x}_{0}\right)+\left(\mathbf{x}-\mathbf{x}_{0}\right)^{T} F_{g_{0}}+\frac{1}{2}\left(\mathbf{x}-\mathbf{x}_{0}\right)^{T} F^{2} g_{0}(\mathbf{x}-\mathbf{x}(8)$

where $F_{80}$ and $F^{2} g_{0}$ denote the gradient vector and the Hessian matrix at $\mathbf{x}_{0}$. The second-order structures of local intensity variations around each point of a volume can be described by the original intensity, the gradient vector, and the Hessian matrix. In this paper, we use the Hessian matrix to measure the $3 \mathrm{D}$ edge structure.

The Hessian matrix is given by

$$
\nabla^{2} g=\left[\begin{array}{lll}
g_{x x} & g_{x y} & g_{x z} \\
g_{y x} & g_{y z} & g_{y z} \\
g_{z x} & g_{z y} & g_{z z}
\end{array}\right]
$$

where the partial second-order differentiation is interpreted as a convolution with derivatives of Gaussians:

$$
\begin{gathered}
g_{x x}=\frac{\partial}{\partial x}\left(\sigma_{1}^{2} g(x) * \frac{\partial}{\partial x} G\left(x, \sigma_{1}\right)\right) \\
G\left(x, \sigma_{1}\right)=\frac{1}{\sqrt{2 \pi \sigma_{1}^{2}}} e^{-\frac{\|x\|^{2}}{2 \sigma_{1}^{2}}}
\end{gathered}
$$

where $\sigma_{1}$ is a Gaussian parameter which controls the response of the derivative function to the specific local tumor structures and $\delta$ is a parameter. Since the tumor is anomalous, tumor structures can exist at various scales. In order to make filter responses tunable to a width of interest, the Hessian matrix is combined with Gaussian convolution. By adjusting the standard deviation of Gaussian convolution, local structures with a specific range of widths can be enhanced. The filter responses decrease as $\sigma_{1}$ increases unless appropriate normalization is performed. Let the eigenvalues of $\nabla^{2} g$ be $\alpha_{1}, \alpha_{2}, \alpha_{3} \quad\left(\alpha_{1} \geq \alpha_{2} \geq \alpha_{9}\right)$. $\alpha_{1}$ gives the maximum second-derivative value. The eigenvalues specify the blob-like local structures. The $3 \mathrm{D}$ derivative cost is defined as follows.

$F_{u}=\lambda_{2}\left|\alpha_{1}\right| \cdot \tau_{\max }$

where $\lambda_{2}$ is the scaling parameter. $T_{\max }$ is the maximum region cost allowed.

\section{3) Downhill term}

The novel downhill cost which is integrated into the energy function is based on the analysis of tumor activity on PET. On FDG-PET images, the lesions with high metabolism have high uptake, so the tumor is clearly identified. However, the transition from the tumor SUV to the background SUV is gradual. Thus, the SUV value of a homogeneous tumor is considered as 3D decreasing monotonically [33]. It means the further a voxel from the maximum intensity site of the tumor, the lower the SUV value. So if a voxel has a high SUV value and keeps a constrained distance from the maximum point, the possibility that it belongs to the tumor region is higher. The downhill region is defined as $V_{D}$ :

$$
F_{D}=\left\{\begin{array}{c}
\text { un if } S U F(u) \geq \mu S U F_{m a x} \text { and } d \propto d_{0} \\
d_{0}=\left\|x_{u}-x_{\max }\right\| \text { if } S U F(w) \propto \mu S U F_{m a x}
\end{array}\right\}
$$

The downhill cost is as follows:

$$
D_{u}= \begin{cases}\lambda_{g} T_{\max } & \text { u } \mathbf{E} \mathbb{V}_{V} \\ 0 & \text { atherwise }\end{cases}
$$

where $\mathrm{x}_{\mathrm{max}}$ is the coordinate of the voxel with maximum SUV value within the tumor domain, $x_{l}$, the coordinate of a voxel $u$. $\|$ is the Euclidean distance. $\lambda_{2}, \mu$ is the given parameter. The downhill region effectively extracts the liver, heart and the ambiguous area. Another advantage is that the 
voxels in the exterior of the downhill region can be considered as background seeds for CT segmentation. Fig.6(b) shows one slice with the detected downhill region.

\section{Algorithm 1. Downhill region detection}

Input: PET image and the segmentation result produced by random walk

Output: downhill region image

Begin

1)Within the tumor area which is specified by random walk, we find the voxel with maximum SUV in PET images

2)Starting at the maximum voxel, it searches the neighboring voxels, if the SUV value of the voxel is less than the thresholds, the distance from the current point to the maximum point is computed, denoted by $d_{0}$. if $\operatorname{SUV}(u) \times \mu S U F_{\text {onax }}, d_{0}=\left\|x_{u}-x_{\text {mox }}\right\|$

3)If the voxel satisfies the following condition, then it is specified as the points in the downhill region. if $S U F(w)>\mu S U F_{\text {wax }}$ and $d \propto d_{0}, d \in F_{D}$ End Output the detected downhill region.

\section{4) Boundary term}

The boundary term measures the penalty of assigning different labels to the neighboring voxels $u$ and $v$. We employ a gradient-based cost which has a similar form as the well-known graph cut method [5]. The boundary cost is described as following:

$$
B_{w v}=-\lambda_{q} \log \left(1-\exp \left(\frac{-\left|\nabla G_{R E F}\right|^{2}(u, v)}{2 \sigma_{g}^{2}}\right)\right)
$$

where $\left|\mathbb{F} G_{\mathrm{PEF}}\right|^{2}(w, v)$ denotes the squared gradient magnitude between $u$ and $v, \sigma_{g}$ is a given Gaussian parameter. $\lambda_{4}$ is the scaling coefficient.

\section{Cost function for CT}

The energy function for $\mathrm{CT}$ comprises a region term $R_{u^{s}}\left(f_{u^{c}}\right)$, a boundary term $B_{u^{t} v^{s}}\left(f_{\left.u^{s}, f_{v^{c}}\right)}\right)$ and a shape penalty term $S_{u^{s}}\left(f_{u^{c}}\right)$. The region term and the boundary term are derived from conventional graph cut energy term. The shape term is independent of image information. CT energy function has the following formulation.

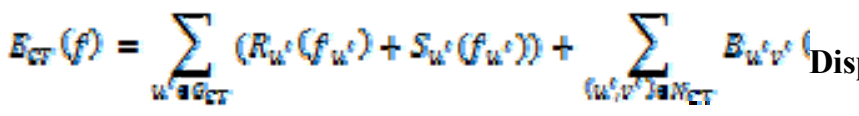

\section{1) Region term}

The hard region cost has the same form as that defined for PET. If voxel $u^{t}$ is in the interior of the pre-defined tumor region, $R_{u^{s}}\left(f_{u^{s}}=1\right)=0$ and $R_{u^{s}}\left(f_{u^{s}}=0\right)=+\infty$. The background seed set is determined by the exterior area of the downhill region which we introduce in Section B.II.(4). Similarly, $R_{u^{t}}\left(f_{u^{s}}=0\right)=0$ and $R_{u^{t}}\left(f_{u^{s}}=1\right)=+\infty$ are defined for every voxel in the background set. For the area

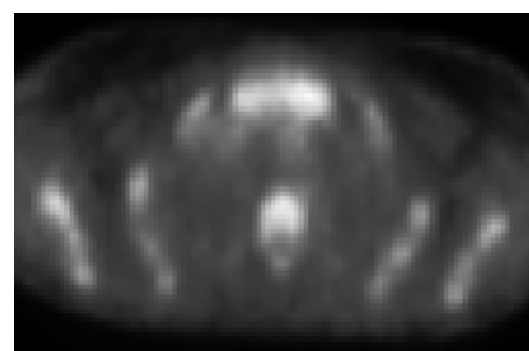

(a)

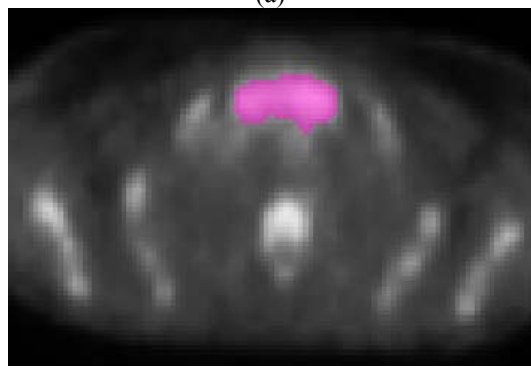

(b)

Fig. 6. one example slice of a downhill region image (a) original PET image (b) downhill region image overlaid on the original PET image. The region in pink is detected by the downhill function. It effectively extracted the spine field which has a similar intensity to the tumor. The region outside the detected downhill region is labeled as the background seed set.

outside the background and foreground fields, the intensity distribution of CT image is assumed as the Gaussian Mixture Model. Instead of being hard constraints, object seed sets are also used to compute the parameter of the Gaussian model. The mean intensity value of all voxels in pre-defined object is denoted by $\bar{g}$ and the corresponding standard deviation is $\sigma$.

$$
R_{u^{i}}\left(f_{u^{i}}=1\right)=-\lambda \xi \log P^{p}\left(\varphi_{u^{c}} \mid f_{u^{i}}=1\right) \propto \frac{\left(8 u^{c}-\bar{g}\right)}{\sigma^{2}}
$$

$$
R_{u^{s}}\left(f_{u^{s}}=0\right)=-\lambda_{s} \log P\left(g u^{s} \mid f_{u^{s}}=0\right) \propto-\log (1-\exp (\text { Dis }
$$

where $\lambda_{5}, \lambda_{6}$ is the scaling parameter.

\section{2) Shape term}

The shape term [34] is independent of image information. The region recognized by random walk is used as the shape model to locate the tumor site. The basic idea of the shape penalty term is to consider the distance from the current voxel to the model area as the penalty of assigning label $f=1$ to voxel, the larger the distance, the larger the cost. The shape constraint function has the following form.

$$
S_{u^{c}}\left(f_{u^{c}}\right)=\lambda_{\tau}\left(1-\exp \left(-\frac{d\left(u^{s}, x_{Q}\right)}{\eta_{v}}\right)\right)
$$

where $d\left(u^{T}, x_{0}\right)$ is the distance from voxel $u^{r}$ to the current shape $x_{0}$ which is identified by random walk,$\lambda$, is the constant parameter. If $u^{r}$ is inside object $0, d\left(u^{T} x_{0}\right)=0$, otherwise, $d\left(u^{7}, x_{0}\right)$ equals to the Euclidian distance from voxel $u^{r}$ to the center of the shape $x_{0} \cdot r_{b}$ is the radius of a circle. Fig.7(a) shows an example slice of the cost image for region term. 


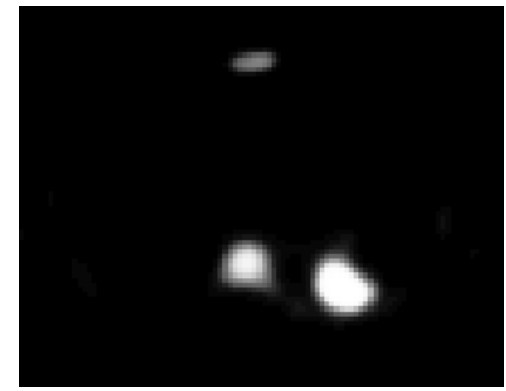

(a)

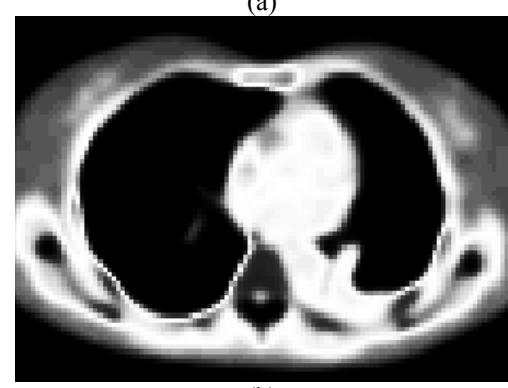

(b)

Fig. 7. one example slice of a cost image for region term in (a) PET image (b) CT image

\section{3) Boundary term}

A gradient based function similar to that defined for PET is utilized, which takes the form

$$
B_{u^{s} v^{c}}=-\operatorname{Ag} \log \left(1-\exp \left(\frac{-\left|\nabla G_{\mathrm{Cr}}\right|^{2}\left(u^{s}, v^{r}\right)}{2 \sigma_{g^{s}}{ }^{2}}\right)\right)
$$

where $\left|\nabla G_{S T}\right|^{2}\left(u^{r}, v^{r}\right)$ denotes the squared gradient magnitude between $u^{r}$ and $v^{r}, \sigma_{g^{r}}$ and $\lambda_{6}$ are given parameters. that just encloses the object. $\lambda_{g}$ is the scaling constant.

\section{Context function}

To make the best incorporation of the information in PET and $\mathrm{CT}$, a context term is introduced. The context energy function takes the form as follows [ ]:

$$
E_{\operatorname{context}}(f)=p\left(1-\mid N_{u}-N_{u} t\right)+q
$$

where $p$ is a scaling constant, $q$ is the penalty for an inconsistent segmentation. $N_{u}$ and $N_{u^{s}}$ are the normalization of the region cost in PET and CT images. The cost is normalized between $[0,1]$. The normalization is to make sure that if the two voxels in PET and CT images belong to the same label set (either to foreground or background), they will have similar region cost. If a pair voxel $(u, u$ ') has similar region cost, a larger context cost will be assigned to penalize the inconsistency between PET and CT. The context cost enables our method to follow the prominent features form each modality and obtain two different volumes on PET and CT.

\section{EXPERIMENTS VALIDATION}

In this section, we applied our proposed algorithm upon the. Our method segments two tumor volumes from PET and CT images.

In this subsection, the effectiveness of the proposed algorithm in delineating uptake regions from PET images was shown. The proposed method was carried out on 18 sets of PET-CT images for validation. For quantitative assessment, the segmentation performance on PET images was evaluated by comparing the computed results against the reference standard. The reference standard is produced by averaging from the two manual contours delineated by experts. In this work, we only use the segmentation results on PET for quantitative validation.

\section{A. Datasets}

Our co-segmentation approach was evaluated in a data set which consists of 183 -D FDG PET-CT images obtained from a dual PET and CT scanner. These 18 sets are from different patients with non-small cell lung cancer (NSCLC). For each slice of PET images, the matrix size is $92 \times 92$ with a voxel size $5.47 \times 5.47 \times 3.27 m^{2}$. For CT image, the matrix size is $312 \times 312$ with a voxel size $0.98 \times 0.98 \times 3.27 n m n^{2}$. PET will be registered with $\mathrm{CT}$ image to obtain one-to-one node correspondence. The Gross Tumor Volume (GTV) was obtained by two experts manually on the PET images by the guidance of the corresponding CT image.

\section{B. Initialization}

In the preprocessing step, registration and initialization are required. Affine transform is used to register PET image with CT image to obtain one-to-one voxel correspondence. As random walk is an interactive method, user defines seeds of the object and background for guidance of the desired content to be extracted. Since random walk doesn't have small cut problem, a small number of seeds also works on the method. The flowchart of the proposed algorithm is shown in Fig.2. First, anatomical and functional image are filtered by gradient anisotropic diffusion filter which is used to smooth images but preserve the edge. Then random walk is conducted on PET images and the initial results are obtained. Downhill region is detected on PET images and the area outside the downhill region is used as background seed set for CT segmentation. PET energy and CT energy and context cost is computed, encoded on the constructed graph. The entire energy is minimized by solving a max-flow/min-cut problem in the constructed graph in a polynomial time.

\section{Parameter setting}

In the experiments, the parameter was empirically employed for all analyzed datasets. For random walk segmentation, the weighting parameter $\beta$ is set as 40 . For the segmentation on PET, we set the maximum region cost as $T_{\max }=100000$, indicating that if the possibility a voxel belongs to the desired area is very small, the cost of labeling it as the target region is very large. In PET segmentation, the regional term plays a more important role than the boundary term so that we set $\lambda_{1}=1$ for data term. The parameter for the $3 \mathrm{D}$ derivative $\cos t \lambda_{2}$ is set as 0.1 . The factor for the downhill term is set as $\lambda_{2}=0.1$. For the smoothness term, $\lambda_{4}=0.4, \sigma_{g}=0.5$. For the CT segmentation, 


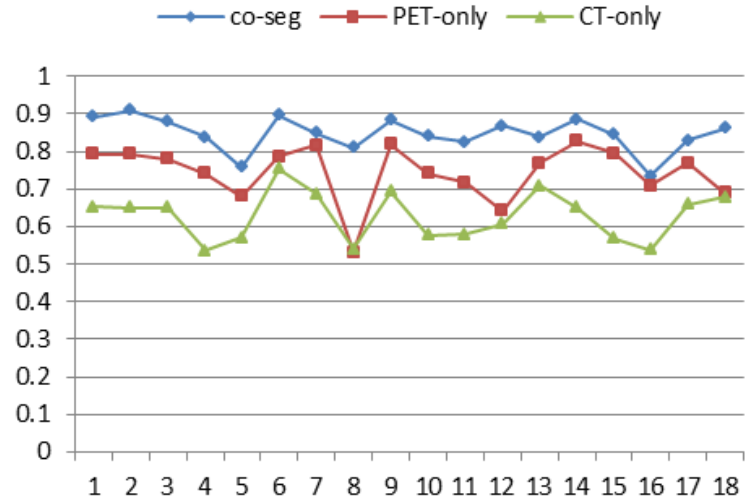

(a)

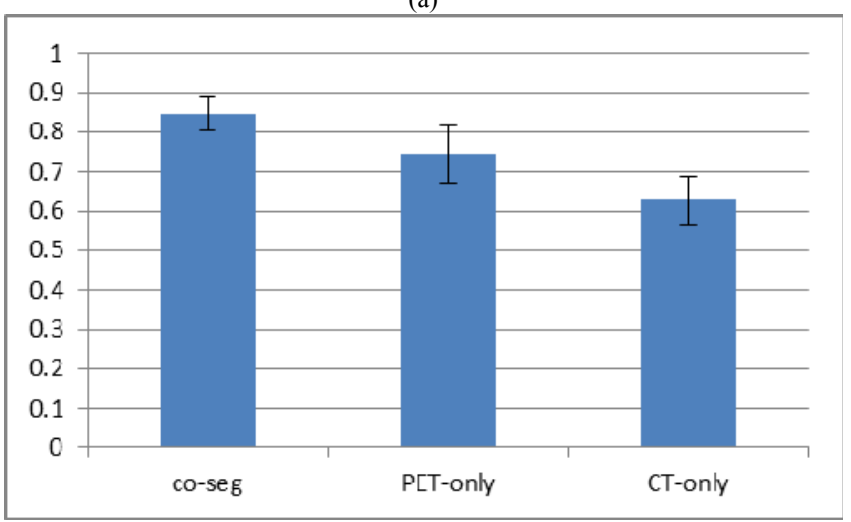

(b)

Fig. 8. Quantitative results and comparative performance evaluation based on the computed DSC values. (a) DSC values (b) mean DSC and the standard deviantion of the three methods.

the boundary term has a great impact on delineated results, thus we set $\lambda_{\mathrm{g}}=\lambda_{\mathrm{s}}=1 \mathrm{~g}$ for the regional term and the coefficient for shape term $\lambda_{5}=0.1$. For the boundary term, $\lambda_{8}=5$, $\sigma_{g}=0.5$. In the context cost function, the scaling coefficient was set as $\mathrm{p}=0.2$, the inconsistent penalty was set as $q=0.1$.

\section{Evaluation of PET Segmentation}

\section{1) Evaluation metrics}

To measure the segmentation performance, Dice Similarity Coefficient (DSC) was used to evaluate the accuracy of the segmented result against the ground truth. DSC measures how the segmented volume matches the reference standard. We denote the segmented results and the ground truth by $U_{1}$ and $U_{2}$. The Dice Similarity Coefficient is computed as:

$$
\operatorname{DSC}\left(U_{1}, U_{2}\right)=2 \cdot \frac{\left|U_{1} \cap U_{2}\right|}{\left|U_{1}\right|+\left|U_{2}\right|}
$$

\section{2) Comparison with graph cut solely on PET or CT}

To determine the performance of co-segmentation of PET and CT images, we compare the proposed method with graph cut solely conducted on PET or CT images. The three methods are all applied to the 18 datasets with identical initialization. The cost functions in the three methods have the same parameter setting. DSC is computed to provide quantitative analysis.

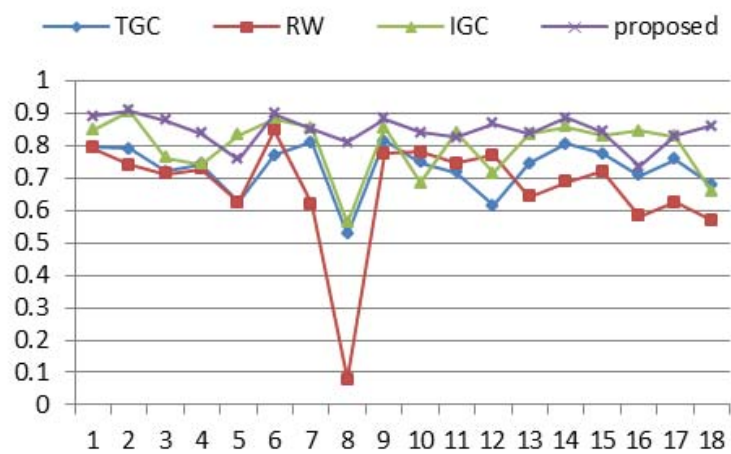

(a)

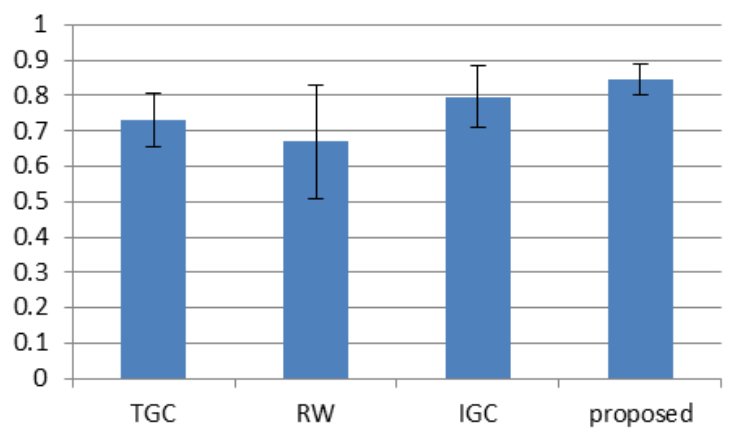

(b)

Fig. 9. Quantitative results and comparative performance evaluation based on the computed DSC values. (a) DSC values (b) mean DSC and the standard deviantion of the three methods.

TABLE I

QUANTITATIVE DSC RATE USING THE PROPOSED METHOD COMPARED WITH FIVE OTHER METHODS: GRAPH UCT SOLELY

USING PET, GRAPH CUT SOLELY USING CT, RANDOM WALK METHOD, IMPROVED GRAPH CUT, TRADITIONAL GRAPH CUT. RESULTS ARE REPORTED AS MEAN \pm STANDARD DEVIATION

\begin{tabular}{|l|l|c|}
\hline Rank & Methods & DSC \\
\hline 1 & Our method & $0.847 \pm 0.045$ \\
\hline 2 & PET only & $0.744 \pm 0.072$ \\
\hline 3 & CT only & $0.628 \pm 0.063$ \\
\hline 4 & RW & $0.670 \pm 0.162$ \\
\hline 5 & TGC & $0.730 \pm 0.075$ \\
\hline 6 & IGC & $0.797 \pm 0.088$ \\
\hline
\end{tabular}

\section{3) Comparison with other methods}

In this paper, graph cut and random walk are combined together and devised a novel energy function for both PET and CT. To evaluate the combination significance of the two methods, we compare the proposed method with random walk (RW) conducted solely on PET images and improved graph cut co-segmentation method which has the novel energy regularization but without the random walk method (denoted by IGC). To evaluate the effectiveness of the energy representation incorporated into the conventional functions, our algorithm is compared with the traditional graph cut co-segmentation method (TGC) in which only the conventional energy functions are utilized to adjust to the limited features of images. In this comparison, the same foreground and background seed sets are used as initialization. 


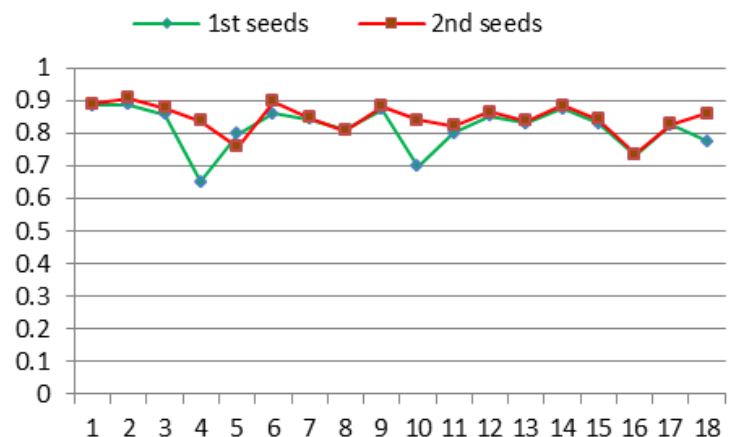

Fig.10. Quantitative results for the proposed method using two different initialization, measured by DSC metrics.

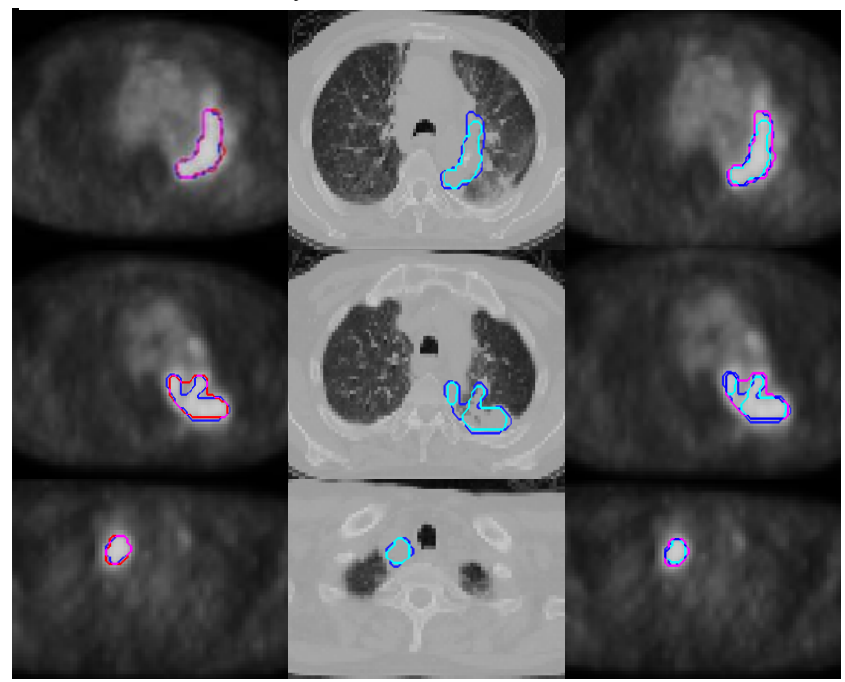

Fig. 11. Three typical segmentation examples are shown in each column. First column: our proposed co-segmentation results on PET (red) and ground truth (green) are overlaid. Second column: co-segmentation from CT (red) and ground truth (green). Third column: all segmentations and ground truth are overlaid on the PET images.

\section{4) Robustness to seed Initialization}

To assess the reliability of the proposed method to the location and quantity of the seed points, the observer was asked to do the second initialization. The second initialization was conducted with the information from the first initialization and intended to change the location of seed points and decrease $10 \%$ of the seeds quantity. All 18 datasets were experimented using the second initialization. The DSC scores were computed to evaluate the stability of the proposed method to seeds.

\section{E. Results}

The DSC rates were computed and compared amongst the four methods. Fig.9 exhibits the performance comparison among the proposed method and the other methods. The figure demonstrates that the proposed method has consistent performance for all 18 datasets. And quantitative evaluation of our method in comparisons indicates that the delineation volumes obtained from the proposed algorithm have higher accuracy than the graph cut method solely using the PET or CT.

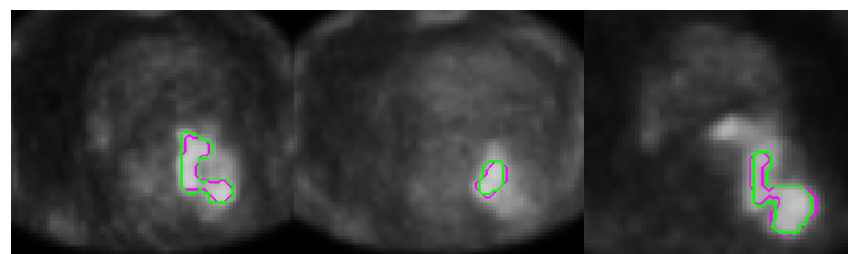

(a) (b) (c)

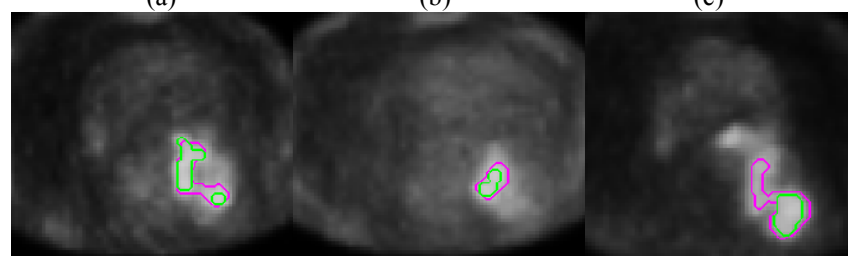

(d) (e) (f)

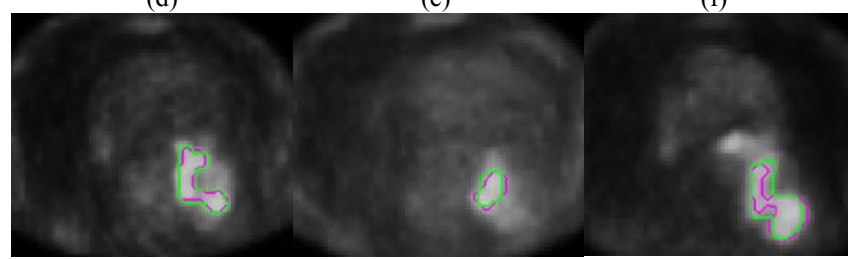

(g) (h) (i)

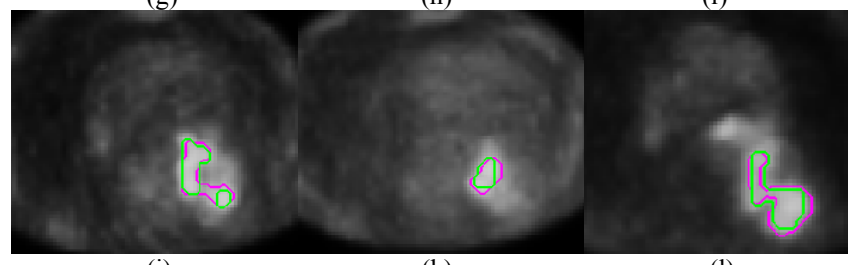

(j) (k) (1)

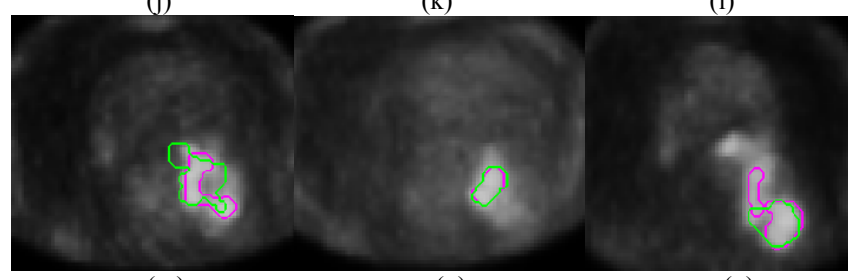

(m)

(n)

(o)

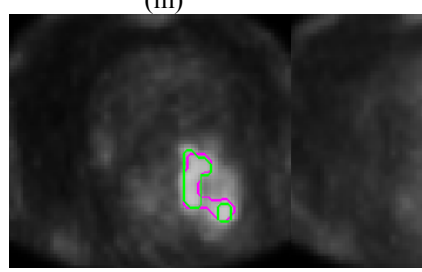

(p)

(q)

(r)

Fig. 12. Three different comparative segmentation results of lesions are shown in each column. (a)-(c) segmentation results conducted by traditional co-segmentation graph cut method. (d)-(f) segmentation results by our proposed method. (g)-(i) segmentation results by random walk (j)-(l)ground truth. (m)-(o) segmentation results which is conducted solely on CT images (p)-(r) the results by graph cut conducted solely on PET images.

\section{DISCUSSION}

The proposed method combines two popular graph-based methods, random walk and graph cut method to segment tumor simultaneously on PET and CT. The novel feature lies on the problem-specific objective function and the combination of the two graph-based algorithms. 


\section{A. Significance of the combination of random walk and graph cut.}

In the graph cut method, a common problem is the small cut behavior since the method tries to minimize the total energy. In this paper, we use random walk as an initial processor to provide the prior tumor contours which are used as the seed points for graph cut. The integration of random walk effectively avoids the small cut problem of graph cut. Compared to graph cut method, random walk needs a smaller number of seeds which reduces user's intervention. It is also able to accommodate the uncertainties in the seed initialization. Since random walk has advantage in capturing weak object boundaries, the combination makes it significant in dealing with fuzzy boundaries of tumor.

\section{B. Significance of the novel function}

In this paper, graph cut method is improved by the introduction of the novel energy function. The downhill function takes the advantage of the feature of the tumor intensity distribution and the distance to the tumor site. This function helps to locate the tumor and extract the surrounding tissues which have similar intensity distribution to tumor. The 3D derivative feature utilizes the largest eigenvalue of the Hessian matrix to represent the salient character of PET images. CT lacks metabolism information and has low contrast, so that it is difficult to distinguish between the disease areas and the normal tissues. To locate the tumor site in CT image, we introduce a shape constraint function which utilizes the prior information of the tumor as a shape model to constrain the tumor region growing. This function translates the Euclidean distance to the cost, the large the distance from the voxel to the shape model, the large the cost.

\section{Possible improvement}

The identification of the foreground and background seed points can be automated. Ulas et al. [3] have developed a seed automatic detection algorithm. The algorithm proposed in [3] set a threshold for the PET SUV value to define the object seeds first. Then neighboring voxels are searched to find voxels with SUV values lower than another threshold. Those voxels are defined as background seeds. However, the heart and liver with high uptake SUV value which is similar to the tumor intensity will be wrong detected as the foreground seeds, leading to a wrong guidance to the segmentation. In the future, the detailed information of the lung structure will be extracted from PET and CT images. The location and the size of the organs are contained in the structure information. This structure information will facilitate the identification of the foreground seeds and do not yield ambiguity.

Multi-modality has become a standard method in clinical pathological analysis, each kind of the imaging techniques capture different characteristics of human body. Although the co-segmentation makes use of the superior tumor contrast from PET images and the exquisite anatomical information from CT images, PET and CT cannot provide enough complementary inform to each other. In the future, we plan to apply our framework to joint segmentation of MRI, PET and CT. The combination of the three modalities, and even more modalities, will produce a more accurate segmentation tumor results.
In this study, the parameters for the weighting function were set empirically. In the future, we will try to employ learning methods to find appropriate factor on the training datasets.

\section{CONCLUSION}

In this paper, we aim to segment the tumor simultaneously on PET and CT by effectively integrating the physiological information from PET and anatomical information from CT. The key novelty of our method is the incorporation of random walk and graph cut method and the cost function we design for graph cut segmentation on PET and CT. The integration of the two methods can avoid the small cut problem in graph cut and has good performance in capturing fuzzy boundary of the tumor on PET images. The novel energy function we proposed effectively utilizes the features from both PET and CT images. Each feature has contributions to tumor segmentation. The SUV distribution feature makes our method perform well on segmentation of both homogeneous and heterogeneous tumor. The downhill feature can extract the surrounding tissues with high uptakes similar to tumor SUV and its exterior can be used as the background seeds for CT segmentation. The 3D local structure enhancement feature has the advantage of specifying the blob-like tumor objects. The shape term in the energy function for CT segmentation helps locate the tumor site. The results demonstrated the effectiveness of the proposed novel energy functions when compared our method to the traditional graph cut method. The significant improvement is achieved when compared to other segmentation methods.

\section{ACKNOWLEDGMENT}

This research is support by the Medical Image Processing, Analysis and Visualization Lab. We acknowledge the First Affiliated Hospital of Soochow University who supports us the lung cancer dataset.

\section{REFERENCES}

[1] J. Bradley, W. Thorstad, S. Mutic, T. Miller, F. Dehdashti, B. Siegel, W. Bosch, and R. Bertrand, "Impactof FDG-PET on radiation therapy volume delineation in non-small-cell lung cancer,'Int. J. Radiat. Oncol., Biol. Phys., vol. 59, no. 1, pp. 78-86, 2004.

[2] Davis, J., Reiner, B., Huser, M., Burger, C., Szekely, G., Ciernik, I., 2006. Assessment of $18 \mathrm{f}$ pet signals for automatic target volume definition in radiotherapy treatment planning. Radiotherapy and Oncology 80, 43-50.

[3] Erdi, Y., Rosenzweig, K., Erdi, A., Macapinlac, H., Hu, Y., Braban, L., Humm, J., Squire, O., Chui, C., Larson, S., et al., 2002. Radiotherapy treatment planning for patients with non-small cell lung cancer using positron emission tomography (pet). Radiotherapy and Oncology 62, 51-60.

[4] Boellaard, R., Krak, N., Hoekstra, O., Lammertsma, A., 2004. Effects of noise, image resolution, and ROI definition on the accuracy of standard uptake values: a simulation study. Journal of Nuclear Medicine 45, 1519-1527.

[5] A.Baardwijk,G.Bosmans,L.Boersma,J.Buijsen,S.Wanders,M.Hochste nbag, R. Suylen, A. Dekker, C. Dehing-Oberije, R. Houben, S. Bentzen, M. Kroonenburgh, P. Lambin, and D. Ruysscher, "PET-CTbased auto-contouring in non-small-cell lung cancer correlates with pathology and reduces interobserver variability in the delineation of the primary tumor and involved nodal volumes,"Int. J. Radiat. Oncol., Biol. Phys., vol. 68, no. 3, pp. 771-778, 2007 
[6] R. J. Steenbakkers, J. C. Duppen, I. Fitton, K. E. Deurloo, L. J. Zijp, E. F. Comans, A. L. Uitterhoeve, P. T. Rodrigus, G. W. Kramer, J. Bussink, K.D.Jaeger, J.S.Belderbos，P.J.Nowak，C.R.v.Herk， and M.Rasch, "Reduction of observer variation using matched CT-PET for lung cancer delineation: A three-dimensional analysis," Int. J. Radiat. Oncol., Biol., Phys., vol. 64, no. 2, pp. 435-48, Mar. 2006

[7] Erasmus, J., Gladish, G., Broemeling, L., Sabloff, B., Truong, M., Herbst, R., Munden, R., 2003. Interobserver and intraobserver variability in measurement of non-smallcell carcinoma lung lesions: implications for assessment of tumor response. Journal of Clinical Oncology 21, 2574-2582

[8] J.L.Fox, R.Rengan, W.O'Meara, E.Yorke, Y.Erdi, S.Nehmeh, S. A. Leibel, and K. E. Rosenzweig, "Does registration of PET and planning CT images decrease interobserver and intraobserver variation in delineating tumor volumes for non-small-cell lung cancer?,” Int. J. Radiat. Oncol., Biol., Phys., vol. 62, no. 1, pp. 70-5, May 2005

[9] M.T.Munley, L.B.Marks, C.Scarfone, G.S.Sibley, E.F.Patz,T. G. Turkington, R. J. Jaszczak, D. R. Gilland, M. S. Anscher, and R. E. Coleman, "Multimodality nuclear medicine imaging in three-dimensional radiation treatment planning for lung cancer: Challenges and prospects," Lung Cancer, vol. 23, no. 2, pp. 105-14, Feb. 1999

[10] B. Ulas, K. Jayaram, "Joint segmentation of anatomical and functional images: Application in quantification of lesions from PET, PET-CT, MRI-PET, and MRI-PET-CT," Medical Image Analysis, vol.17, pp.929-945, 2013.

[11] Bagci, U., Udupa, J., Yao, J., Mollura, D., 2012b. Co-segmentation of functional and anatomical images. In: Proc. of Medical Image Computing and Computer Assisted Intervention, pp. 459-467

[12] Han D.F., Bayouth J., Song Q., Taurani A., Sonka, M., Buatti J., Wu X, "Globally optimal tumor segmentation in PET-CT images: A graph-based co-segmentation method," in Information Processing in Medical Imaging. Springer Berlin Heidelberg, 2011, pp.245-256

[13] Xiuying Wang, Cherry Ballangan, Hui Cui, "Lung Tumor Delineation Based on Novel Tumor-Background Likelihood Models in PET-CT Images" IEEE Transactions on Nuclear Science, vol.61, no.1, FEB., 2014.

[14] Song Qi, Bai Junjie, Han Dongfeng, Bhatia S., Sun Wenqing, Rockey W., Wu Xiaodong, "Optimal co-segmentation of tumor in PET-CT images with context information," IEEE Transactions on Medical Imaging, vol.32, no.9, pp.1685-1697, 2013

[15] Boykov Yuri.Y., and M.P.Jolly., "Interactive graph cuts for optimal boundary \& region segmentation of objects in ND images," in IEEE International Conference on Computer Vision, 2001, pp. 105-112.

[16] Yuri Boykov, Olga Veksler, "Graph Cuts in Vision and Graphics: Theories and Applications" Handbook of Mathematical Models in Computer Vision, Springer,2006.

[17] Yuri Boykov, Vladimir Kolomogorov, "An Experimental Comparison of Min-Cut/Max-Flow Algorithms for Energy Minimization in Vision" IEEE Transactions on Pattern Analysis and Machine Intelligence, vol.28, no.9, Sep. 2004

[18] Yuri Boykov Olga Veksler Ramin Zabih "Fast Approximate Energy Minimization via Graph Cuts" IEEE Transactions on Pattern Analysis and Machine Intelligence, vol. 23, no.11, NOV., 2001

[19] Y. Boykov and G. Funka-Lea, "Graph cuts and efficient N-D image segmentation,'Int. J. Comput. Vis., vol. 70, no. 2, pp. 109-131, 2006.

[20] Leo Grady, "Random Walks for Image Segmentation," IEEE Transactions on Pattern Analysis and machine Intelligence, vol. 28, no. 11, pp. 1768-1783, Nov. 2006

[21] Leo Grady, Thomas Schiwietz, and Shmuel Aharon, "Random Walks for Interactive Organ Segmentation in Two and Three Dimensions: Implementation and Validation" the $8^{\text {th }}$ International Conference on
Medical Image Computing and Computer Assisted Intervention, Palm Springs,CA.

[22] Ali Kemal Sinop, Leo Grady, "A Seeded Image Segmentation Framework Unifying Graph Cuts And Random Walker Which Yields A New Algorithm" published in Proc. Of ICCV 2007, Rio de Janeiro, Brazil, Oct., 2007

[23] Y. E. Erdi, O. Mawlawi, S. M. Larson, M. Imbriaco, H. Yeung, R. Finn, and J. L. Humm, "Segmentation of lung lesion volume by adaptive positron emission tomography image thresholding,"Cancer, vol. 80, pp. 2505-2509, 1997

[24] Drever, L., Roa, W., McEwan, A., Robinson, D., 2007. Iterative threshold segmentation for pet target volume delineation. Medical Physics 34, 1253

[25] Brambilla, M., Matheoud, R., Secco, C., Loi, G., Krengli, M., Inglese, E., 2008. Threshold segmentation for pet target volume delineation in radiation treatment planning: the role of target-to-background ratio and target size. Medical Physics 35, 1207.

[26] Zaidi, H., El Naqa, I., 2010. Pet-guided delineation of radiation therapy treatment volumes: a survey of image segmentation techniques. European Journal of Nuclear Medicine and Molecular Imaging 37, 2165-2187.

[27] Zaidi, H., Diaz-Gomez, M., Boudraa, A., Slosman, D., 2002. Fuzzy clustering-based segmented attenuation correction in whole-body pet imaging. Physics in Medicine and Biology 47, 1143.

[28] Hatt, M., Cheze le Rest, C., Turzo, A., Roux, C., Visvikis, D., 2009. A fuzzy locally adaptive bayesian segmentation approach for volume determination in pet. IEEE Transactions on Medical Imaging 28, $881-893$.

[29] Jentzen, W., Freudenberg, L., Eising, E., Heinze, M., Brandau, W., Bockisch, A., 2007. Segmentation of pet volumes by iterative image thresholding. Journal of Nuclear Medicine 48, 108-114.

[30] Bagci, U., Yao, J., Caban, J., Turkbey, E., Aras, O., Mollura, D., 2011. "A graph-theoretic approach for segmentation of pet images," In: Engineering in Medicine and Biology Society, EMBS, 2011 Annual International Conference of the IEEE, pp. 8479-8482.

[31] Ballangan C., Wang X., Fulham M., Eberl S., \& Feng D. D., "Lung tumor segmentation in PET images using graph cuts" Computer Methods and Programs in Biomedicine, vol. 109, pp. 260-268, 2013.

[32] Ballangan, Cherry and Wang, Xiuying and Fulham, Michael and Eberl, Stefan and Yin, Yong and Feng, Dagan (2011)Automated Delineation of Lung Tumors in PET Images Based on Monotonicity and a Tumor-Customized Criterion. IEEE Transactions on Information Technology in Biomedicine, 15 (5). pp. 691-702. ISSN 1089-7771

[33] Yoshinobu Sato, Carl-Fredrik Westin, Abhir Bhalerao, Shin Nakajima, Nobuyuki Shiraga, Shinichi Tamura, and Ron Kikinis "Tissue Classification Based on 3D Local Intensity Structures for Volume Rendering," IEEE Transactions on Visualization and Computer Graphics, vol.6, no.2, APRIL JUNE, 2000

[34] Chen Xinjian, Udupa J.K., Bagci U., Zhuge Y., Yao J., "Medical image segmentation by combining graph cuts and oriented active appearance models," IEEE Transactions on Image Processing, vol.21, no.4, pp.2035-2046, 2012.

[35] Qi Song Mingqing Chen Junjie Bai, Milan Sonka and Xiaodong Wu "Surface-Region Context in Optimal Multi-Object Graph-based Segmentation: Robust Delineation of Pulmonary Tumors" This work was supported in part by NSF grants CCF-0830402 and CCF-0844765, and NIH grants R01-EB004640 and K25-CA123112.

[36] Kang Li, Xiaodong Wu, Danny Z. Chen, Milan Sonka, "Optimal Surface segmentation in Volumetric Images A Graph-Theoretic Approach" IEEE Transactions on Pattern Analysis and Machine Intelligence, vol.28, no. 1, Jan. 2006. 